\title{
The Color Accuracy of the Kubelka-Munk Theory for Various Colorants in Maxillofacial Prosthetic Material*
}

T. MA, W. M. JOHNSTON ${ }^{1,3}$, and A. KORAN, III ${ }^{2}$

Department of Fixed Prosthodontics. School of Dentistry, University of the Pacific, San Francisco, California $94115 ;{ }^{\prime}$ Section of Restorative and Prosthetic Dentistry, College of Dentistry, The Ohio State University. Columbus, Ohio 43210; and ${ }^{2}$ Department of Biomaterials, School of Dentistry, The University of Michigan, Ann Arbor, Michigan 48109

\begin{abstract}
The reflectance model developed by Kubelka and Munk was evaluated for agreement in color prediction of thick pigmented samples and for linearity of optical absorption and scattering coefficients with concentration of colorant in maxillofacial elastomer. The colorants tested were generic opacifiers, dry mineral earth pigments, and fibrous colorants. Significant linear relationships were commonly found between the optical coefficients and the concentration of the colorants. These relationships indicated occasional optical interaction between the colorants and the elastomer. Color differences between theoretical and observed colors of the thick samples averaged $2.96,3.47$, and 1.60 for the opacifiers, mineral earth pigments, and fibrous colorants, respectively, when measured using the CIELAB uniform-color space. The agreement between theoretical and observed colors was significantly closer for the fibrous colorants than for the dry mineral earth pigments of the same labeled color.
\end{abstract}

\section{J Dent Res 66(9):1438-1444, September, 1987}

\section{Introduction.}

The solution of an optical reflectance theory by Kubelka and Munk (1931) and its subsequent simplification by Kubelka (1948) gave rise to the application of this theory to describe the color and translucency of colored materials within the paint (Billmeyer and Abrams, 1973) and plastic (Billmeyer and Abrams, 1972) industries. Judd (1937) initially applied the Kubelka-Munk theory to dental materials, and O'Brien and his co-workers have advanced this theory in its application to dental composite (Miagawa et al., 1981) and porcelain materials (Woolsey et al., 1984; O’Brien et al., 1985).

Kubelka and Munk (1931) solved the simultaneous flow of two diffuse light fluxes (forward and backward) within a turbid medium in optical contact with a reflective backing. An assumption involved in the development of the Kubelka-Munk theory is that no reflections occur at the interfacial boundaries between the turbid material and the air at the front surface, or between the turbid material and the backing. Corrections for the reflections which do occur have been presented by Duntley (1942) and Saunderson (1942), but the value for internal reflection proposed by Mudgett and Richards (1973) enables these corrections to provide better agreement between observed reflectance and the Kubelka-Munk theory (Johnston et al., 1986).

The Kubelka-Munk theory, corrected as above, and the linear dependence of the optical absorption and scattering coefficients upon colorant concentration have provided the basis for colorant formulation for materials in general (Johnston, 1973; Kuehni, 1975), but Kuehni (1975) warns of possible

Received for publication October 20, 1986

Accepted for publication March 6, 1987

* Based in part on a thesis submitted in partial fulfillment of the requirements for the degree of Master of Science (Dental Materials) at The University of Michigan. This study was supported in part by USPHS Research Grant R01-DE04130 and Research Grant R01DE05423.

${ }^{3}$ To whom correspondence and reprint requests should be addressed optical or mechanical interactions of the coloring pigments and the substrate. However, Billmeyer and Richards (1973) state that, for translucent plastics, "two-flux turbid-medium theory is inadequate.... Four-flux theory is adequate ... but better accuracy can be obtained by the use of six-flux or many-flux calculations". Theories involving more than two diffuse light fluxes incorporate collimated light fluxes at specific angles to interfacial boundaries of the turbid medium. The purposes of this study were to ascertain the ability of the Kubelka-Munk theory to describe the color induced by different types of colorants in maxillofacial elastomer and to study the influence of concentrations of these colorants.

\section{Materials and methods.}

Three categories of colorants were chosen for this study: six generic opacifiers, nine dry mineral earth pigments (Artskin Products Co., 200 Circle E Bldg., Military Circle, Norfolk, VA), and six colored nylon fibrous colorants known as "flockings'" (Claremont Flock Corp., Claremont, NH). The generic opacifiers were titanium dioxide (Batch 33578, J.T. Baker Co., Phillipsburg, NJ), tin (IV) oxide (Batch 2332, Allied Chemical \& Dye Corp., New York, NY), zinc oxide (manufacturer's No. 8832, batch BVY, Mallinckrodt, Inc., St. Louis, MO), kaolin (manufacturer's No. G-1001, Factor II Products, Lakeside, AZ), zinc stearate (Batch 41585, Merck \& Co., Inc., Rahway, NJ), and talc (Batch 23652, Johnson's Baby Products Co., Skillman, NJ). The mineral earth pigments were labeled black, white, red, blue, dark buff, light brown, medium brown, red brown, and yellow. The flockings were labeled blue, white, tan, brown, red brown, and red.

For the purpose of determining the optical absorption and scattering coefficients, the opacifiers and the mineral earth pigments were separately incorporated into $30 \mathrm{~g}$ of medical-grade elastomer (Silastic MDX4-4210 silicone elastomer, Lot $\mathrm{HH}$ 062180, Dow Corning Corp., Medical Products Div., Midland, MI 48640) in concentrations of $0.1,0.05$, and $0.02 \mathrm{wt} \%$. The flockings were separately incorporated into the silicone base at concentrations of 2, 1, and $0.5 \mathrm{wt} \%$. To the colored silicone base was then added $10 \mathrm{wt} \%$ curing agent (MDX44210 curing agent, Lot HH-033211, Dow Corning Corp., Medical Products Div., Midland, MI 48640). The mix was then placed under reduced atmospheric pressure in order to eliminate incorporated air bubbles, and was poured into an aluminum mold which produced specimens $60 \mathrm{~mm}$ by 120 $\mathrm{mm}$, with a thickness within $0.25 \mathrm{~mm}$ of $1.25 \mathrm{~mm}$. The mold and elastomer were returned to reduced pressure and cured at $65^{\circ} \mathrm{C}$ for eight hours. Two specimens of each concentration of each colorant and two specimens of unpigmented elastomer were fabricated, making a total of 128 specimens for optical absorption and scattering determination.

In addition, thicker specimens were formed for each colorant at the greatest concentration given above. These specimens were formed in an identical manner, except that $60 \mathrm{~g}$ of elastomer base was used, and the molds produced specimens 40 
$\mathrm{mm}$ in diameter and $40 \mathrm{~mm}$ in height. Twenty-one specimens were formed for the determination of reflectivity, which is the reflectance at infinite optical thickness.

Titanium oxide and black dry mineral earth colorants were chosen for the fabrication of the dark and light backings to be used for optical coefficient determinations. One percent by weight of each of these two colorants was used separately to make backings as described above for the thick reflectance specimens.

The optical coefficients were determined by the methods fully described by Woolsey et al. (1984) and by Johnston et al. (1986), with one exception: No custom-made sample port plate was used, since the elastomeric specimens were of sufficient size to be held within the standard sample port. Reflectance measurements were made with illumination beam condensers in place, such that any reflected light scattered toward the edge of the sample would be included in the measurement. To summarize the determination method, the reflectance spectrum of each thin specimen was measured with the specimen in optical contact with each of the two backings, and the Kubelka-Munk absorption coefficient, $\mathrm{K}$, and scattering coefficient, $S$, were calculated by the solution of two conditions of the Kubelka-Munk theory. For each concentration of each colorant and for the unpigmented material, two determinations of the optical coefficient were obtained and averaged at every wavelength.

The linear relationship between the optical coefficients of each colorant and the colorant concentration were analyzed by linear regression analysis at wavelengths of 450,500 , and 650 $\mathrm{nm}$. The optical coefficients of the unpigmented elastomer were also included.

The reflectivity was calculated by the equation of Kubelka (1948):

$$
\operatorname{Rinf}=\left[1+K / S+\left(K^{2} / S^{2}+2 K / S\right)^{1 / 2}\right]^{-1}
$$

where $\mathrm{K}$ and $\mathrm{S}$ are the average optical absorption and scattering coefficients, respectively. This equation was corrected for interfacial reflections (Duntley, 1942; Saunderson, 1942) using the value of internal reflection proposed by Mudgett and Richards (1973). The calculated color parameters were determined by the method of C.I.E. (1976, 1985). The observed reflectivity for the 21 thick specimens was measured on a diffuse reflectance spectrophotometer (Beckman Acta-C3 UV-visible spectrophotometer with ASPH-U integrating sphere, Beckman Instruments, Inc., Irvine, CA 92664). The observed color parameters were determined from the average of two measurements of reflectivity. The color difference between the calculated and observed color parameters was then determined within the (L*, a*, b*)-uniform color space (C.I.E., 1976). Statistical analysis of the color differences was performed for those mineral earth and flocking colorants which had identical color names.

\section{Results.}

In general, the absorption spectra of the chromatic colorants exhibited absorption maxima at the wavelengths corresponding to the opposite color of each colorant studied. For example, Fig. I displays the spectral optical absorption coefficients of the unpigmented elastomer and of the elastomer with the three concentrations of red flockings. Fig. 2 similarly displays the spectral scattering coefficients of yellow dry mineral earth pigment.

The results of all linear regression analyses of the optical absorption (K) and scattering (S) coefficients are presented in Tables 1 and 2, respectively. The intercept is the regression

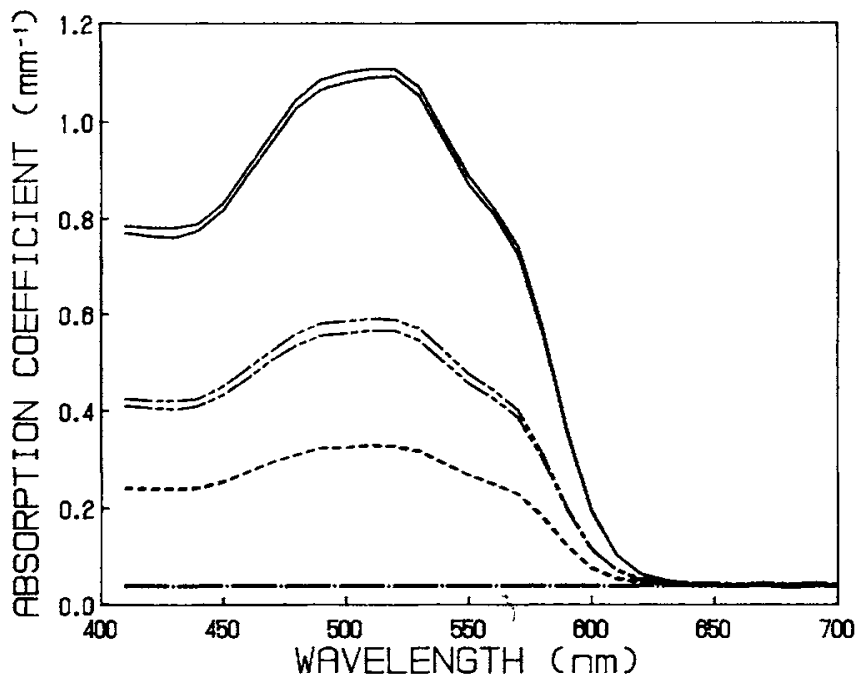

Fig. 1 - Spectra of optical absorption coefficients for maxillofacial elastomer with red flocking in concentrations of $2.0 \mathrm{wt} \%$ (solid line), 1.0 wt $\%$ (long-short-short dashed line), $0.5 \mathrm{wt} \%$ (dashed line), and $0.0 \mathrm{wt} \%$ (dash-dot line).

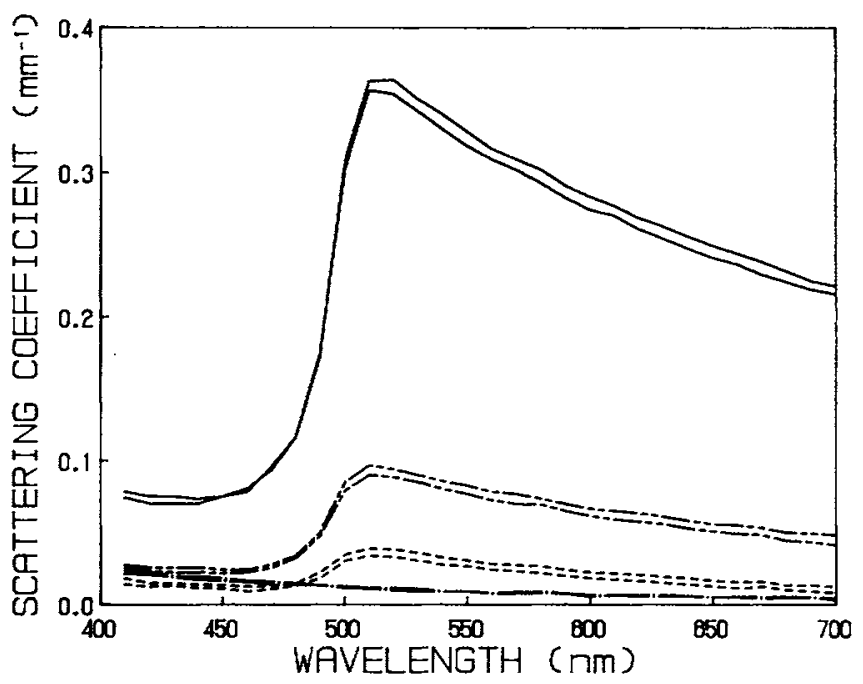

Fig. 2 - Spectra of optical scattering coefficients for maxillofacial elastomer with yellow dry mineral earth pigment in concentrations of 0.10 wt $\%$ (solid), $0.05 \mathrm{wt} \%$ (long-short-short dashed line), $0.02 \mathrm{wt} \%$ (dashed line), and $0.00 \mathrm{wt} \%$ (dash-dot line).

estimate of each coefficient for unpigmented elastomer, and the slope represents the increase in coefficient per increase in concentration in wt \%. The average optical absorption coefficients for unpigmented elastomer were $0.039,0.038$, and 0.038 $\mathrm{mm}^{-1}$ at the wavelengths of 450,500 , and $650 \mathrm{~nm}$, respectively. The average optical scattering coefficients for unpigmented elastomer were $0.018,0.009$, and $0.005 \mathrm{~mm}^{-1}$ at the wavelengths of 450,500 , and $650 \mathrm{~nm}$, respectively. The standard error is that of the regression for each coefficient at the average concentration studied. Since there were 126 regression analyses, only those with a statistical probability of 0.0003 or less were considered statistically significant. Figs. 3 through 6 present the values of selected optical coefficients and show the positions of the linear regression lines relative to the observed optical coefficients.

Fig. 7 presents the calculated and observed reflectivities for 0.1 wt\% dark buff dry mineral earth colorant. Table 3 gives the color differences between calculated and observed color 
TABLE 1

LINEAR REGRESSION ANALYSES OF OPTICAL ABSORPTION COEFFICIENT (K) VERSUS COLORANT CONCENTRATION

\begin{tabular}{|c|c|c|c|c|c|}
\hline Colorant & $\begin{array}{c}\text { Wavelength } \\
(\mathrm{nm})\end{array}$ & $\begin{array}{l}\text { Intercept } \\
\text { (per } \mathrm{mm} \text { ) }\end{array}$ & $\begin{array}{c}\text { Slope } \\
\text { (per mm) }\end{array}$ & $\begin{array}{c}\text { Standard } \\
\text { Error }\end{array}$ & $\mathrm{P}$ \\
\hline Titanium Dioxide & $\begin{array}{l}450 \\
550 \\
650\end{array}$ & $\begin{array}{l}0.033 \\
0.033 \\
0.034\end{array}$ & $\begin{array}{l}-20 \\
-26 \\
-31\end{array}$ & $\begin{array}{l}0.0077 \\
0.0063 \\
0.0049\end{array}$ & $\begin{array}{l}0.0337 \\
0.0045 \\
0.0005\end{array}$ \\
\hline Tin (IV) Oxide & $\begin{array}{l}450 \\
550 \\
650\end{array}$ & $\begin{array}{l}0.040 \\
0.040 \\
0.041\end{array}$ & $\begin{array}{l}-16 \\
-17 \\
-15\end{array}$ & $\begin{array}{l}0.0019 \\
0.0024 \\
0.0025\end{array}$ & $\begin{array}{l}0.0001 \\
0.0003 \\
0.0007\end{array}$ \\
\hline Zinc Oxide & $\begin{array}{l}450 \\
550 \\
650\end{array}$ & $\begin{array}{l}0.040 \\
0.041 \\
0.041\end{array}$ & $\begin{array}{l}-22 \\
-20 \\
-18\end{array}$ & $\begin{array}{l}0.0021 \\
0.0025 \\
0.0029\end{array}$ & $\begin{array}{r}<0.0001 \\
0.0001 \\
0.0005\end{array}$ \\
\hline Kaolin & $\begin{array}{l}450 \\
550 \\
650\end{array}$ & $\begin{array}{l}0.039 \\
0.038 \\
0.039\end{array}$ & $\begin{array}{l}5.5 \\
5.3 \\
4.2\end{array}$ & $\begin{array}{l}0.0022 \\
0.0019 \\
0.0018\end{array}$ & $\begin{array}{l}0.0369 \\
0.0240 \\
0.0498\end{array}$ \\
\hline Zinc Stearate & $\begin{array}{l}450 \\
550 \\
650\end{array}$ & $\begin{array}{l}0.038 \\
0.038 \\
0.039\end{array}$ & $\begin{array}{l}2.2 \\
1.3 \\
0.7\end{array}$ & $\begin{array}{l}0.0019 \\
0.0016 \\
0.0015\end{array}$ & $\begin{array}{l}0.2647 \\
0.4112 \\
0.6600\end{array}$ \\
\hline Talc & $\begin{array}{l}450 \\
550 \\
650\end{array}$ & $\begin{array}{l}0.038 \\
0.038 \\
0.038\end{array}$ & $\begin{array}{l}2.4 \\
3.0 \\
2.0\end{array}$ & $\begin{array}{l}0.0019 \\
0.0016 \\
0.0013\end{array}$ & $\begin{array}{l}0.2330 \\
0.0584 \\
0.1466\end{array}$ \\
\hline Red Artskin & $\begin{array}{l}450 \\
550 \\
650\end{array}$ & $\begin{array}{l}0.053 \\
0.050 \\
0.032\end{array}$ & $\begin{array}{r}462 \\
442 \\
-12\end{array}$ & $\begin{array}{l}0.0146 \\
0.0123 \\
0.0014\end{array}$ & $\begin{array}{r}<0.0001 \\
<0.0001 \\
0.0001\end{array}$ \\
\hline Med. Brown Artskin & $\begin{array}{l}450 \\
550 \\
650\end{array}$ & $\begin{array}{l}0.051 \\
0.045 \\
0.040\end{array}$ & $\begin{array}{r}361 \\
289 \\
82\end{array}$ & $\begin{array}{l}0.0114 \\
0.0064 \\
0.0016\end{array}$ & $\begin{array}{l}<0.0001 \\
<0.0001 \\
<0.0001\end{array}$ \\
\hline Black Artskin & $\begin{array}{l}450 \\
550 \\
650\end{array}$ & $\begin{array}{l}0.042 \\
0.039 \\
0.036\end{array}$ & $\begin{array}{l}1154 \\
1174 \\
1219\end{array}$ & $\begin{array}{l}0.0073 \\
0.0068 \\
0.0058\end{array}$ & $\begin{array}{l}<0.0001 \\
<0.0001 \\
<0.0001\end{array}$ \\
\hline White Artskin & $\begin{array}{l}450 \\
550 \\
650\end{array}$ & $\begin{array}{l}0.039 \\
0.040 \\
0.041\end{array}$ & $\begin{array}{l}-23 \\
-20 \\
-16\end{array}$ & $\begin{array}{l}0.0035 \\
0.0026 \\
0.0027\end{array}$ & $\begin{array}{l}0.0004 \\
0.0002 \\
0.0007\end{array}$ \\
\hline Light Brown Artskin & $\begin{array}{l}450 \\
550 \\
650\end{array}$ & $\begin{array}{l}0.041 \\
0.039 \\
0.041\end{array}$ & $\begin{array}{l}288 \\
123 \\
6.7\end{array}$ & $\begin{array}{l}0.0046 \\
0.0020 \\
0.0025\end{array}$ & $\begin{array}{r}<0.0001 \\
<0.0001 \\
0.0300\end{array}$ \\
\hline Dark Buff Artskin & $\begin{array}{l}450 \\
550 \\
650\end{array}$ & $\begin{array}{l}0.036 \\
0.036 \\
0.040\end{array}$ & $\begin{array}{r}470 \\
71 \\
-22\end{array}$ & $\begin{array}{l}0.0045 \\
0.0023 \\
0.0012\end{array}$ & $\begin{array}{l}<0.0001 \\
<0.0001 \\
<0.0001\end{array}$ \\
\hline Blue Artskin & $\begin{array}{l}450 \\
550 \\
650\end{array}$ & $\begin{array}{l}0.041 \\
0.038 \\
0.038\end{array}$ & $\begin{array}{r}12 \\
238 \\
186\end{array}$ & $\begin{array}{l}0.0026 \\
0.0038 \\
0.0027\end{array}$ & $\begin{array}{r}0.0031 \\
<0.0001 \\
<0.0001\end{array}$ \\
\hline Red Brown Artskin & $\begin{array}{l}450 \\
550 \\
650\end{array}$ & $\begin{array}{l}0.034 \\
0.033 \\
0.034\end{array}$ & $\begin{array}{r}291 \\
243 \\
77\end{array}$ & $\begin{array}{l}0.0066 \\
0.0066 \\
0.0042\end{array}$ & $\begin{array}{l}<0.0001 \\
<0.0001 \\
<0.0001\end{array}$ \\
\hline Yellow Artskin & $\begin{array}{l}450 \\
550 \\
650\end{array}$ & $\begin{array}{l}0.026 \\
0.032 \\
0.033\end{array}$ & $\begin{array}{r}1240 \\
-23 \\
-23\end{array}$ & $\begin{array}{l}0.0651 \\
0.0063 \\
0.0055\end{array}$ & $\begin{array}{r}<0.0001 \\
0.0091 \\
0.0012\end{array}$ \\
\hline Blue Flocking & $\begin{array}{l}450 \\
550 \\
650\end{array}$ & $\begin{array}{l}0.050 \\
0.056 \\
0.053\end{array}$ & $\begin{array}{l}32 \\
49 \\
43\end{array}$ & $\begin{array}{l}0.0113 \\
0.0184 \\
0.0152\end{array}$ & $\begin{array}{l}<0.0001 \\
<0.0001 \\
<0.0001\end{array}$ \\
\hline White Flocking & $\begin{array}{l}450 \\
550 \\
650\end{array}$ & $\begin{array}{l}0.034 \\
0.034 \\
0.035\end{array}$ & $\begin{array}{l}0.25 \\
0.23 \\
0.24\end{array}$ & $\begin{array}{l}0.0054 \\
0.0040 \\
0.0036\end{array}$ & $\begin{array}{l}0.3774 \\
0.2783 \\
0.2089\end{array}$ \\
\hline Tan Flocking & $\begin{array}{l}450 \\
550 \\
650\end{array}$ & $\begin{array}{l}0.049 \\
0.043 \\
0.042\end{array}$ & $\begin{array}{l}11 \\
5.8 \\
2.3\end{array}$ & $\begin{array}{l}0.0098 \\
0.0045 \\
0.0032\end{array}$ & $\begin{array}{l}<0.0001 \\
<0.0001 \\
<0.0001\end{array}$ \\
\hline Brown Flocking & $\begin{array}{l}450 \\
550 \\
650\end{array}$ & $\begin{array}{l}0.058 \\
0.050 \\
0.043\end{array}$ & $\begin{array}{l}28 \\
21 \\
10\end{array}$ & $\begin{array}{l}0.0199 \\
0.0130 \\
0.0052\end{array}$ & $\begin{array}{l}<0.0001 \\
<0.0001 \\
<0.0001\end{array}$ \\
\hline Red Brown Flocking & $\begin{array}{l}450 \\
550 \\
650\end{array}$ & $\begin{array}{l}0.056 \\
0.051 \\
0.042\end{array}$ & $\begin{array}{l}40 \\
34 \\
16\end{array}$ & $\begin{array}{l}0.0195 \\
0.0141 \\
0.0048\end{array}$ & $\begin{array}{l}<0.0001 \\
<0.0001 \\
<0.0001\end{array}$ \\
\hline Red Flocking & $\begin{array}{l}450 \\
550 \\
650\end{array}$ & $\begin{array}{l}0.048 \\
0.047 \\
0.041\end{array}$ & $\begin{array}{l}39 \\
42 \\
0.03\end{array}$ & $\begin{array}{l}0.0108 \\
0.0112 \\
0.0026\end{array}$ & $\begin{array}{r}<0.0001 \\
0.0002 \\
0.8331\end{array}$ \\
\hline
\end{tabular}


TABLE 2

LINEAR REGRESSION ANALYSES OF OPTICAL SCATTERING COEFFICIENT (S) VERSUS COLORANT CONCENTRATION

\begin{tabular}{|c|c|c|c|c|c|}
\hline Colorant & $\begin{array}{l}\text { Wavelength } \\
\text { (nm) }\end{array}$ & $\begin{array}{l}\text { Intercept } \\
\text { (per mm) }\end{array}$ & $\begin{array}{c}\text { Slope } \\
\text { (per mm) }\end{array}$ & $\begin{array}{c}\text { Standard } \\
\text { Error }\end{array}$ & $\mathbf{P}$ \\
\hline Titanium Dioxide & $\begin{array}{l}450 \\
550 \\
650\end{array}$ & $\begin{array}{r}-0.159 \\
-0.020 \\
0.021\end{array}$ & $\begin{array}{l}610 \\
504 \\
417\end{array}$ & $\begin{array}{l}0.0314 \\
0.0274 \\
0.0238\end{array}$ & $\begin{array}{l}<0.0001 \\
<0.0001 \\
<0.0001\end{array}$ \\
\hline Tin (IV) Oxide & $\begin{array}{l}450 \\
550 \\
650\end{array}$ & $\begin{array}{l}0.016 \\
0.007 \\
0.004\end{array}$ & $\begin{array}{l}74 \\
55 \\
44\end{array}$ & $\begin{array}{l}0.0032 \\
0.0026 \\
0.0019\end{array}$ & $\begin{array}{l}<0.0001 \\
<0.0001 \\
<0.0001\end{array}$ \\
\hline Zinc Oxide & $\begin{array}{l}450 \\
550 \\
650\end{array}$ & $\begin{array}{l}0.015 \\
0.007 \\
0.004\end{array}$ & $\begin{array}{l}98 \\
63 \\
47\end{array}$ & $\begin{array}{l}0.0033 \\
0.0023 \\
0.0017\end{array}$ & $\begin{array}{l}<0.0001 \\
<0.0001 \\
<0.0001\end{array}$ \\
\hline Kaolin & $\begin{array}{l}450 \\
550 \\
650\end{array}$ & $\begin{array}{l}0.019 \\
0.010 \\
0.006\end{array}$ & $\begin{array}{l}4.7 \\
2.5 \\
2.5\end{array}$ & $\begin{array}{l}0.0009 \\
0.0006 \\
0.0007\end{array}$ & $\begin{array}{l}0.0012 \\
0.0063 \\
0.0087\end{array}$ \\
\hline Zinc Stearate & $\begin{array}{l}450 \\
550 \\
650\end{array}$ & $\begin{array}{l}0.018 \\
0.010 \\
0.005\end{array}$ & $\begin{array}{l}2.9 \\
1.6 \\
2.7\end{array}$ & $\begin{array}{l}0.0009 \\
0.0008 \\
0.0005\end{array}$ & $\begin{array}{l}0.0158 \\
0.0737 \\
0.0012\end{array}$ \\
\hline Talc & $\begin{array}{l}450 \\
550 \\
650\end{array}$ & $\begin{array}{l}0.017 \\
0.009 \\
0.005\end{array}$ & $\begin{array}{l}2.6 \\
1.6 \\
2.0\end{array}$ & $\begin{array}{l}0.0012 \\
0.0003 \\
0.0004\end{array}$ & $\begin{array}{l}0.0556 \\
0.0022 \\
0.0023\end{array}$ \\
\hline Red Artskin & $\begin{array}{l}450 \\
550 \\
650\end{array}$ & $\begin{array}{l}0.019 \\
0.010 \\
0.002\end{array}$ & $\begin{array}{l}8.2 \\
11 \\
99\end{array}$ & $\begin{array}{l}0.0018 \\
0.0014 \\
0.0039\end{array}$ & $\begin{array}{r}0.0028 \\
0.0002 \\
<0.0001\end{array}$ \\
\hline Med. Brown Artskin & $\begin{array}{l}450 \\
550 \\
650\end{array}$ & $\begin{array}{l}0.018 \\
0.010 \\
0.004\end{array}$ & $\begin{array}{l}6.4 \\
16 \\
49\end{array}$ & $\begin{array}{l}0.0008 \\
0.0006 \\
0.0016\end{array}$ & $\begin{array}{r}0.0002 \\
<0.0001 \\
<0.0001\end{array}$ \\
\hline Black Artskin & $\begin{array}{l}450 \\
550 \\
650\end{array}$ & $\begin{array}{l}0.018 \\
0.009 \\
0.006\end{array}$ & $\begin{array}{r}11.1 \\
8.2 \\
9.2\end{array}$ & $\begin{array}{l}0.0013 \\
0.0009 \\
0.0013\end{array}$ & $\begin{array}{l}0.0001 \\
0.0001 \\
0.0003\end{array}$ \\
\hline White Artskin & $\begin{array}{l}450 \\
550 \\
650\end{array}$ & $\begin{array}{l}0.012 \\
0.006 \\
0.003\end{array}$ & $\begin{array}{r}119 \\
66 \\
46\end{array}$ & $\begin{array}{l}0.0056 \\
0.0032 \\
0.0020\end{array}$ & $\begin{array}{l}<0.0001 \\
<0.0001 \\
<0.0001\end{array}$ \\
\hline Light Brown Artskin & $\begin{array}{l}450 \\
550 \\
650\end{array}$ & $\begin{array}{l}0.016 \\
0.007 \\
0.003\end{array}$ & $\begin{array}{l}16 \\
34 \\
42\end{array}$ & $\begin{array}{l}0.0016 \\
0.0023 \\
0.0026\end{array}$ & $\begin{array}{l}<0.0001 \\
<0.0001 \\
<0.0001\end{array}$ \\
\hline Dark Buff Artskin & $\begin{array}{l}450 \\
550 \\
650\end{array}$ & $\begin{array}{r}0.011 \\
-0.002 \\
-0.051\end{array}$ & $\begin{array}{l}113 \\
172 \\
159\end{array}$ & $\begin{array}{l}0.0076 \\
0.0112 \\
0.0103\end{array}$ & $\begin{array}{l}<0.0001 \\
<0.0001 \\
<0.0001\end{array}$ \\
\hline Blue Artskin & $\begin{array}{l}450 \\
550 \\
650\end{array}$ & $\begin{array}{l}0.016 \\
0.008 \\
0.004\end{array}$ & $\begin{array}{c}21 \\
7.0 \\
12\end{array}$ & $\begin{array}{l}0.0017 \\
0.0010 \\
0.0009\end{array}$ & $\begin{array}{r}<0.0001 \\
0.0003 \\
<0.0001\end{array}$ \\
\hline Red Brown Artskin & $\begin{array}{l}450 \\
550 \\
650\end{array}$ & $\begin{array}{l}0.019 \\
0.010 \\
0.005\end{array}$ & $\begin{array}{l}5.6 \\
8.8 \\
33\end{array}$ & $\begin{array}{l}0.0013 \\
0.0012 \\
0.0010\end{array}$ & $\begin{array}{r}0.0043 \\
0.0002 \\
<0.0001\end{array}$ \\
\hline Yellow Artskin & $\begin{array}{l}450 \\
550 \\
650\end{array}$ & $\begin{array}{r}0.006 \\
-0.026 \\
-0.025\end{array}$ & $\begin{array}{r}61 \\
320 \\
246\end{array}$ & $\begin{array}{l}0.0119 \\
0.0416 \\
0.0346\end{array}$ & $\begin{array}{l}0.0016 \\
0.0002 \\
0.0003\end{array}$ \\
\hline Blue Flocking & $\begin{array}{l}450 \\
550 \\
650\end{array}$ & $\begin{array}{l}0.020 \\
0.011 \\
0.007\end{array}$ & $\begin{array}{l}0.72 \\
0.02 \\
0.07\end{array}$ & $\begin{array}{l}0.0025 \\
0.0024 \\
0.0020\end{array}$ & $\begin{array}{l}0.0009 \\
0.8619 \\
0.4727\end{array}$ \\
\hline White Flocking & $\begin{array}{l}450 \\
550 \\
650\end{array}$ & $\begin{array}{l}0.018 \\
0.010 \\
0.006\end{array}$ & $\begin{array}{l}1.7 \\
1.2 \\
1.0\end{array}$ & $\begin{array}{l}0.0016 \\
0.0010 \\
0.0010\end{array}$ & $\begin{array}{l}<0.0001 \\
<0.0001 \\
<0.0001\end{array}$ \\
\hline Tan Flocking & $\begin{array}{l}450 \\
550 \\
650\end{array}$ & $\begin{array}{l}0.017 \\
0.009 \\
0.005\end{array}$ & $\begin{array}{l}0.58 \\
0.49 \\
0.47\end{array}$ & $\begin{array}{l}0.0007 \\
0.0006 \\
0.0004\end{array}$ & $\begin{array}{l}<0.0001 \\
<0.0001 \\
<0.0001\end{array}$ \\
\hline Brown Flocking & $\begin{array}{l}450 \\
550 \\
650\end{array}$ & $\begin{array}{l}0.018 \\
0.010 \\
0.005\end{array}$ & $\begin{array}{l}0.34 \\
0.27 \\
0.43\end{array}$ & $\begin{array}{l}0.0013 \\
0.0009 \\
0.0007\end{array}$ & $\begin{array}{r}0.0020 \\
0.0008 \\
<0.0001\end{array}$ \\
\hline Red Brown Flocking & $\begin{array}{l}450 \\
550 \\
650\end{array}$ & $\begin{array}{l}0.018 \\
0.010 \\
0.005\end{array}$ & $\begin{array}{l}0.85 \\
0.52 \\
0.84\end{array}$ & $\begin{array}{l}0.0011 \\
0.0015 \\
0.0009\end{array}$ & $\begin{array}{r}<0.0001 \\
0.0003 \\
<0.0001\end{array}$ \\
\hline Red Flocking & $\begin{array}{l}450 \\
550 \\
650\end{array}$ & $\begin{array}{l}0.018 \\
0.010 \\
0.004\end{array}$ & $\begin{array}{l}0.60 \\
0.28 \\
1.20\end{array}$ & $\begin{array}{l}0.0009 \\
0.0007 \\
0.0010\end{array}$ & $\begin{array}{r}<0.0001 \\
0.0002 \\
<0.0001\end{array}$ \\
\hline
\end{tabular}




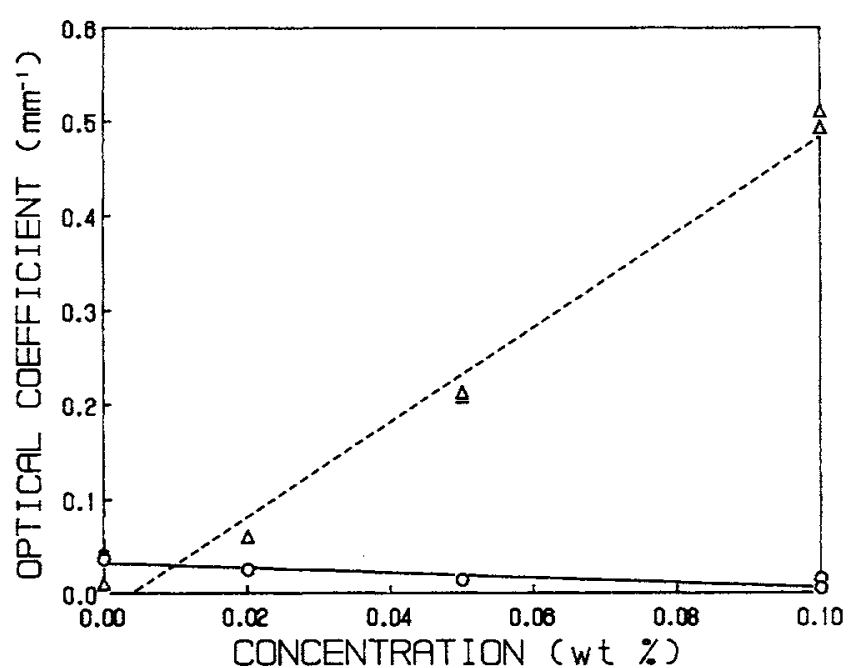

Fig. 3 - Scatter diagrams and regression lines for Kubelka-Munk absorption (open circles and solid line) and scattering (open triangles and dashed line) coefficients $v s$. concentration at $550 \mathrm{~nm}$ for titanium dioxide.

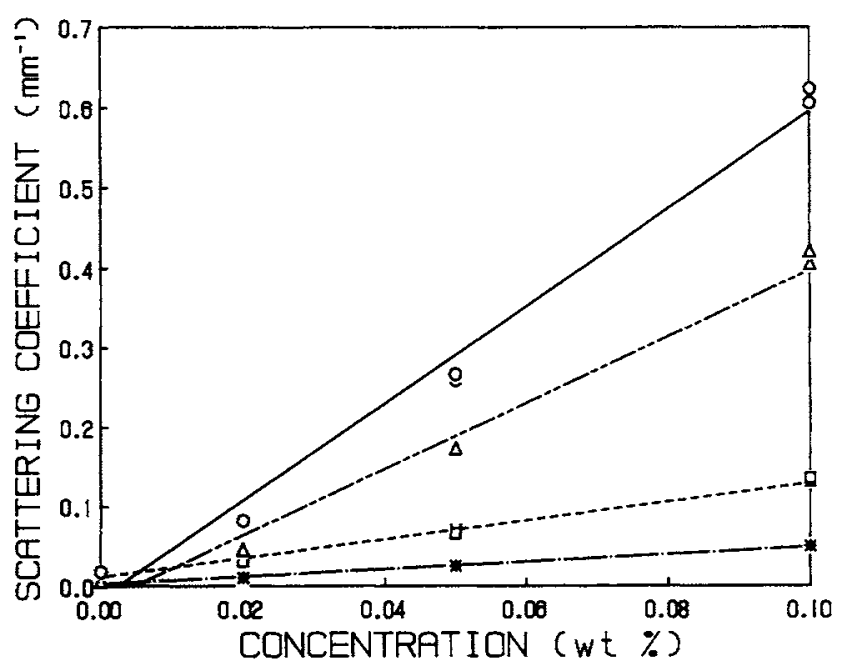

Fig. 4 - Scatter diagrams and regression lines for Kubelka-Munk scattering coefficients $v s$. concentration at 450 and $650 \mathrm{~nm}$ for titanium dioxide (450 nm: open circles and solid line; $650 \mathrm{~nm}$ : open triangles and longshort-short dashed line) and white dry mineral earth pigment (450 nm, open squares and dashed line; $650 \mathrm{~nm}$, * and dash-dot line).

parameters at infinite optical thickness for all colorants studied. These color differences for the mineral earth and flocking colorants of similar color labels are presented in Table 4 with the paired comparison analysis of variance.

\section{Discussion.}

The low error associated with each regression of the optical coefficients upon colorant concentration indicates the consistency with which the Kubelka-Munk theory derives absorption and scattering coefficients relative to the concentrations of many types of colorants within maxillofacial elastomer. Wherever a statistically significant regression was not found, that optical coefficient was not expected to change with concentration for that pigment; e.g., the absorption coefficient for any opacifier may remain at a relatively low value regardless of colorant concentration. The generally consistent values of the intercepts for each optical coefficient indicate the general lack of optical interaction between the maxillofacial elastomer and the color-

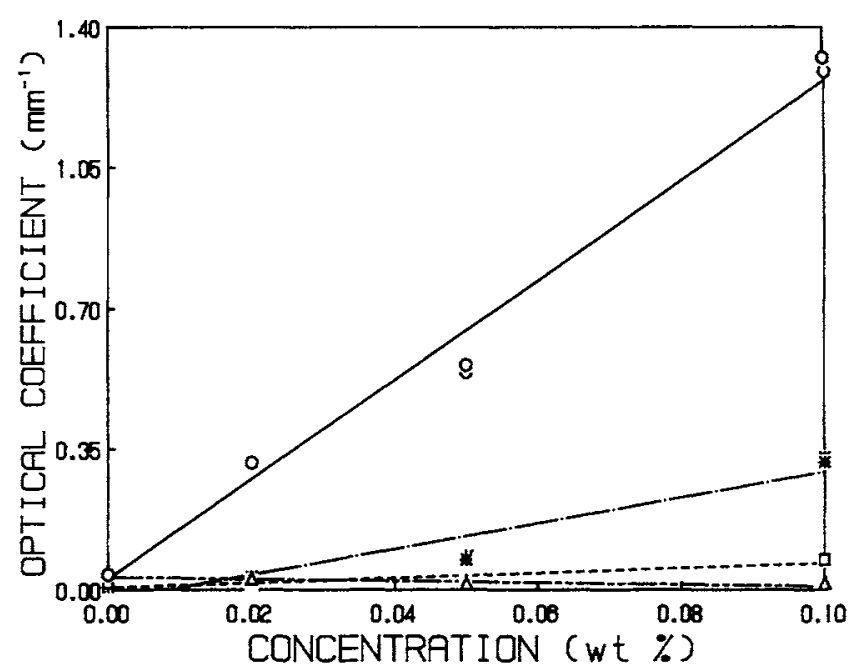

Fig. 5 - Scatter diagrams and regression lines for Kubelka-Munk absorption (K) and scattering (S) coefficients $v s$. concentration at $450 \mathrm{~nm}$ $(\mathrm{K}$, open circles and solid line; $\mathrm{S}$, open squares and dashed line) and 550 $\mathrm{nm}(\mathrm{K}$, open triangles and long-short-short dashed line; $\mathrm{S}, *$ and dash-dot line) for yellow dry mineral earth pigment.

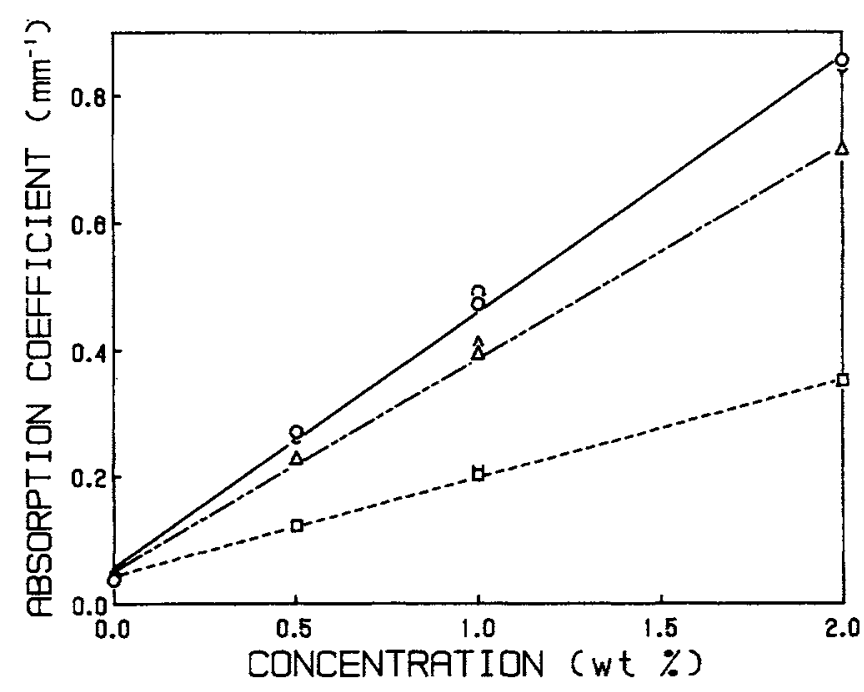

Fig. 6 - Scatter diagrams and regression lines for Kubelka-Munk absorption coefficients $v s$. concentration at 450 (open circles and solid line), 550 (open triangles and long-short-short dashed line), and $650 \mathrm{~nm}$ (open squares and dashed line) for red brown flockings.

ants studied, although specific exceptions are noted, e.g., $\mathrm{K}$ of red and yellow Artskin and brown flocking (Table 1), S of titanium dioxide, dark buff, and yellow Artskin (Table 2). Further study of the size of any porosity in the unpigmented elastomer and the particle size of the colorants is recommended.

The high regression slope for titanium dioxide indicates the superior scattering ability of this colorant. Since a high scattering coefficient is related to high lightness and opacity, titanium dioxide, of all the colorants studied, had the greatest ability to lighten and opacify.

However, the limited general agreement of the observed color with that calculated by the Kubelka-Munk theory indicates that this theory may not always be used to predict the color of pigmented maxillofacial elastomer within a tolerance of even two perceivable color difference units. Despite limitations of this magnitude, the Kubelka-Munk theory has been 


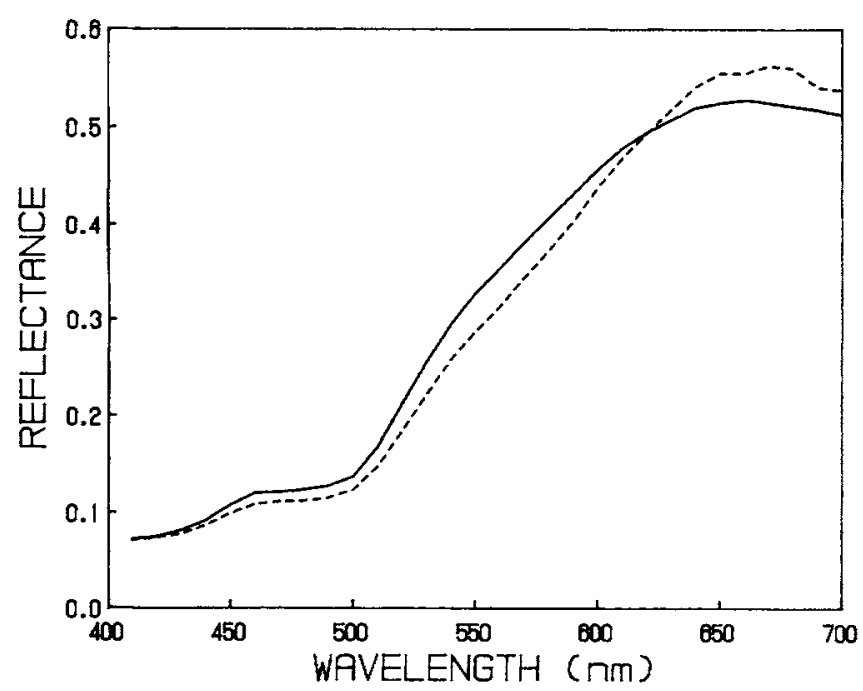

Fig. 7 - Spectra of calculated (dashed line) and observed (solid line) reflectivities for dark buff dry mineral earth pigment at $0.10 \mathrm{wt} \%$.

TABLE 3

COLOR DIFFERENCE BETWEEN OBSERVED AND CALCULATED COLOR PARAMETERS AT INFINITE OPTICAL THICKNESS

\begin{tabular}{ll}
\hline \hline Delta $\mathrm{E}\left(\mathrm{L}^{*}, \mathrm{a}^{*}, \mathrm{~b}^{*}\right)$ & \multicolumn{1}{c}{ Pigment Identification } \\
\hline 2.72 & $0.1 \%$ titanium dioxide \\
2.65 & $0.1 \%$ tin (IV) oxide \\
1.41 & $0.1 \%$ zinc oxide \\
5.31 & $0.1 \%$ kaolin \\
2.68 & $0.1 \%$ zinc stearate \\
3.02 & $0.1 \%$ talc \\
2.96 & Average of six opacifiers \\
2.59 & $0.1 \%$ red Artskin \\
4.30 & $0.1 \%$ med brown Artskin \\
0.11 & $0.1 \%$ black Artskin \\
1.21 & $0.1 \%$ white Artskin \\
4.98 & $0.1 \%$ light brown Artskin \\
4.38 & $0.1 \%$ dark buff Artskin \\
4.74 & $0.1 \%$ blue Artskin \\
3.62 & $0.1 \%$ red brown Artskin \\
5.28 & $0.1 \%$ yellow Artskin \\
3.47 & Average of nine mineral earth colorants \\
0.82 & $2 \%$ blue flocking \\
1.84 & $2 \%$ white flocking \\
3.05 & $2 \%$ tan flocking \\
1.09 & $2 \%$ brown flocking \\
1.16 & $2 \%$ red brown flocking \\
1.67 & $2 \%$ red flocking \\
1.60 & Average of six flocking colorants \\
&
\end{tabular}

promoted as the basis for colorant formulation, since perfect formulations are impossible, and since considerable improvement can be obtained over trial-and-error methods (Johnston, 1973; Kuehni, 1975).

This study also indicates (Table 3 ) that, on the average, the color predicted by the Kubelka-Munk theory was closer to the observed color of a flocking than to that of a mineral earth pigment labeled with the same color. This greater agreement for the fibrous colorant may be due to the more complete elimination of collimated light within the mass of the fiberfilled elastomer, since collimated light is not directly accounted for in the Kubelka-Munk theory. The application of this theory seems based on the assumption that incident collimated illumination is totally and immediately transformed to diffuse light upon entering the turbid medium. The greater volume of the
TABLE 4

MEANS AND STATISTICAL ANALYSIS OF COLOR DIFFERENCES AT INFINITE OPTICAL THICKNESS FOR MINERAL EARTH AND FLOCKING COLORANTS WITH LIKE COLOR LABELING

\begin{tabular}{|c|c|c|c|c|}
\hline \multicolumn{5}{|c|}{ Average Color Difference } \\
\hline Labeled Color & & \multicolumn{3}{|c|}{ Colorant Type } \\
\hline $\begin{array}{l}\text { Blue } \\
\text { White } \\
\text { Red } \\
\text { Light brown or Tan } \\
\text { Medium brown } \\
\text { Red brown }\end{array}$ & & $\begin{array}{l}4.7 \\
1.2 \\
2.5 \\
4.9 \\
4.3 \\
3.6\end{array}$ & & $\begin{array}{l}0.82 \\
1.84 \\
1.67 \\
3.05 \\
1.09 \\
1.16\end{array}$ \\
\hline Average & & 3.5 & & 1.60 \\
\hline \multicolumn{5}{|c|}{ Analysis of Variance } \\
\hline Source of Variation & d.f. & M.S. & F-ratio & Probability \\
\hline $\begin{array}{l}\text { Pigment type } \\
\text { Labeled color } \\
\text { Residual (error) }\end{array}$ & $\begin{array}{l}1 \\
5 \\
5\end{array}$ & $\begin{array}{r}11.638 \\
1.385 \\
1.346\end{array}$ & $\begin{array}{l}8.649 \\
1.029\end{array}$ & $\begin{array}{l}<0.05 \\
>0.25\end{array}$ \\
\hline
\end{tabular}

d.f. $=$ degrees of freedom.

M.S. = mean square

fibrous particles appears to permit most of the incident collimated light to be either scattered or absorbed.

These results support a general lack of perfect agreement of the Kubelka-Munk theory with observed colors, but the relative ease of application of this two-flux reflectance theory is of great importance. Techniques are available for corrections based on a trial coloration and subsequent re-formulation which give negligible color differences between re-formulation and the target color (Kuehni, 1975). The general magnitude of the color differences between the theoretical and observed values supports the further study of this theory for mixtures of colorants within maxillofacial elastomer.

\section{REFERENCES}

BILLMEYER, F.W., Jr. and ABRAMS, R.L. (1972): Predicting Color by the Kubelka-Munk Equations in Opaque and Translucent Systems, Soc Plast Eng Tech Paper 18:117-120.

BILLMEYER, F.W., Jr. and ABRAMS, R.L. (1973): Predicting Reflectance and Color of Paint Films by Kubelka-Munk Analysis. I. Turbid Medium Theory, $J$ Paint Tech 45:23-30.

BILLMEYER, F.W., Jr. and RICHARDS, L.W. (1973): Scattering and Absorption of Radiation by Lighting Materials, $J$ Color Appearance 2:4-15.

C.I.E., COMMISSION INTERNATIONALE de I'ECLAIRAGE (1976): Recommendations on Uniform Colour Spaces, Colour Difference Equations, and Metric Colour Terms, Supplement No. 2 to Publication No. 15, Bureau Central de la CIE, Paris.

C.I.E., COMMISSION INTERNATIONALE de I'ECLAIRAGE (1985): Colorimetry, Official Recommendations of the International Commission on Illumination, 2nd ed., Publication CIE No. 15.2(TC-3.1), Bureau Central de la CIE, Paris.

DUNTLEY, S.Q. (1942): The Optical Properties of Diffusing Materials, $J$ Opt Soc Am 32:61-70.

JOHNSTON, R.M. (1973): Color Theory. In: Pigments Handbook, Vol. 3, T.C. Patton, Ed., New York: Wiley, pp. 229-288.

JOHNSTON, W.M.; O'BRIEN, W.J.; and TIEN, T.-Y. (1986): The Determination of Optical Absorption and Scattering in Translucent Porcelain, Color Res Appl 11:125-130.

JUDD, D.B. (1937): Optical Specification of Light-scattering Materials, Natl Bur Stand J Res 19:287-317.

KUBELKA, P. (1948): New Contributions to the Optics of Intensely Light-scattering Materials. Part I, J Opt Soc 38:448-457. 
KUBELKA, P. and MUNK, F. (1931): Ein Beitrag zur Optik der Farbanstriche, Z Tech Phys 12:593-601.

KUEHNI, R.G. (1975): Computer Colorant Formulation, Lexington, MA: Lexington Books, pp. 11-29, 72-74.

MIAGAWA, Y.; POWERS, J.M.; and O'BRIEN, W.J. (1981): Optical Properties of Direct Restorative Materials, J Dent Res 60:890894.

MUDGETT, P.S. and RICHARDS, L.W. (1973): Kubelka-Munk Scattering and Absorption Coefficients for Use with Glossy, Opaque Objects, J Paint Technol 45:44-53.
O'BRIEN, W.J.; JOHNSTON, W.M.; and FANIAN, F. (1985): Double-layer Color Effects in Porcelain Systems, J Dent Res 64:940943.

SAUNDERSON, J.L. (1942): Calculation of the Color of Pigmented Plastics, $J$ Opt Soc Am 32:727-736.

WOOLSEY, G.D.; JOHNSTON, W.M.; and O'BRIEN, W.J. (1984): Masking Power of Dental Opaque Porcelains, J Dent Res 63:936939 (Erratum, J Dent Res 63:1115). 